CIWA Publishing 2014. The definitive peer-reviewed and edited version of this article is published in the Journal of water and climate change, 5(1), pages 13-24, DOI: 10.2166/wcc.2013.073, and is available from http:// jwcc.iwaponline.com/ Journal of Water and Climate Change, 2013, Vol 5, No 1, pp 13-24

$5 \quad$ Michael Green

6 School Of Applied Sciences, Building 52, Cranfield University, Cranfield, Bedfordshire, England

7 E: m.green@cranfield.ac.uk

$8 \quad \mathrm{~T}:+44(0) 1234758368$

9

10

Prof. Edward Keith Weatherhead (Corresponding Author)

11 School Of Applied Sciences Building 52, Cranfield University, Cranfield, Bedfordshire, England

E: k.weatherhead@cranfield.ac.uk

13 W: http://www.cranfield.ac.uk/sas/aboutus/staff/weatherheadk.html

$14 \mathrm{~T}:+44(0) 1234758368$

\section{irrigation reservoir planning under climate change \\ A critical comparison of using a probabilistic weather generator versus a change factor approach;}


19 Abstract:

In the UK, there is a growing interest in constructing on-farm irrigation reservoirs, however deciding the optimum reservoir capacity is not simple. There are two distinct approaches to generating the future daily weather datasets needed to calculate future irrigation need.

The change factor approach perturbs the observed record using monthly change factors derived from downscaled climate models. This assumes that whilst the climate changes, the day-to-day climate variability itself is stationary. Problems may arise where the instrumental record is insufficient or particularly suspect. Alternatively, probabilistic weather generators can be used to identify options which are considered more robust to climate change uncertainty because they consider non-stationary climate variability.

This paper explores the difference between using the change factor approach and a probabilistic weather generator for informing farm reservoir design at three sites in the UK. Decision outcomes obtained using the current normal practice of $80 \%$ probability of non-exceedance rule and simple economic optimisations are also compared.

Decision outcomes obtained using the change factor approach and probabilistic weather generators are significantly different; whether these differences translate to real-world differences is discussed. This study also found that using the $80 \%$ probability of non-exceedance rule could potentially result in maladaptation. 
Water is integral to the UK's ability to grow high quality horticultural produce. In the UK, approximately 150,000 ha are irrigated during a dry year (Knox et al, 2010). The sustainability of irrigated production is however under threat from competition for water from other sectors, new legislation designed to enhance environmental protection, and climate change (Weatherhead et al, 2008).

Water resources in many catchments are already strained. During summer, many existing water sources become increasingly unreliable and new licenses for summer abstractions are now widely unobtainable or are issued with tight minimum flow or minimum level constraints. Increasingly farmers, agribusiness and water resource managers are being encouraged to build on-farm irrigation reservoirs as part of their water resource strategy to avoid the restrictions and environmental impact of abstraction during summer months (Weatherhead et al, 2008). Climate change is expected to simultaneously increase water demand and reduce water availability (Kang et al, 2009).

The unpredictability of the future climate is perhaps the greatest challenge facing the water industry (Harris et al, 2012). In the UK at least, much of the current infrastructure including irrigation reservoirs were built on the assumption that the climate in which it was built would endure for its entire lifetime - this is no longer the case (Harris et al, 2012).

Two responses have emerged in reaction to the risks posed by future climate change, namely mitigation and adaptation (Füssel, 2007). Mitigation refers to "an anthropogenic intervention to reduce the sources or enhance the sinks of greenhouse gases" (IPCC, 2001). In contrast, adaptation, studied in this paper, refers to "the adjustment in natural or human systems in response to actual or expected climatic stimuli or their effects which moderates harm or exploits beneficial opportunities"

61 (Parry et al, 2007, p.6). In the UK, adaptation planning emerged as a policy issue in 1997 in response to the formulation of the UK Climate Impacts Programme (UKCIP) (Hedger et al, 2006), receiving 
renewed interest with the passing of the Climate Change Act 2008 (Tang and Dessai, 2012). The apparent 'failure' of high profile climate change protocols (e.g. the Kyoto protocol) has undermined confidence in the success of mitigation efforts, making adaptation a more attractive surrogate (Anderson and Bows, 2011; Fung et al, 2011 and Sanderson et al, 2011; Harris et al, 2012).

A number of approaches to adaptation have been identified. Vulnerability-led adaptation includes methods aimed at identifying and reducing present community/system vulnerability; thereby reducing future exposure to potentially damaging impacts. Scenario-led adaptation, studied here, uses future climate change projections to assess future climate change impacts. Downscaled regional-scale climate scenario data can be fed into impact models; the outputs are then used to inform adaptation, to maximise potential benefits and/or minimise potential risks (Wilby and Dessai, 2010). A hybrid approach, combining elements of vulnerability-led and scenario-led approaches has recently emerged, though is not the focus of this paper (Brown and Wilby, 2012).

Scenario-led adaptation is limited by the financial and technical capacity of the individuals undertaking the adaptation; their risk appetite, the availability of high quality downscaled climate change information and the type of adaptation options being considered (Adger et al, 2005; Dessai et al, 2005). Despite greater awareness of its benefits (Füssel, 2007; Ranger et al, 2010), few realworld cases of scenario-led adaptation decisions have been realised (Tompkins et al, 20120), with large sector and regional differences in the type of adaptation considered. This limited uptake has been attributed to a variety of factors; see Moser and Ekstrom (2010) for an extensive discussion.

Scenario-led adaptation is used here to model irrigation demand and inform farm reservoir design in a semi-humid climate. A sufficiently long daily weather record is essential to adequately gauge the amount of water required. For the baseline period (1961-1990), irrigation demand calculations are often based on the observed record, though this may be substituted with a synthetic series from a weather generator provided it has been suitably calibrated (Green and Weatherhead, 2013). Similarly, a sufficiently long record of future daily weather data is required to model irrigation 
demand under the effects of climate change. Future weather data is typically generated from downscaled global climate models (GCM). GCM outputs are often only available as monthly values (Holman et al, 2009), which are generally insufficient for modelling dry year supplemental irrigation demand and many hydrological processes. They can however be used to perturb an observed or synthetic daily series using the 'change factor' approach (Loaiciga et al, 2000), elsewhere referred to as perturbation or the "delta-change" method (Prudhomme et al, 2002). A change factor is obtained for each month in the future series, these figures are then used to perturb an observed baseline daily series to produce a future series i.e. applying a January monthly change factor of $10 \%$ to an observed series would make all of the daily values in the future series for the month of January $+10 \%$ larger (Holman et al, 2009). A criticism of the change factor approach is that it assumes that the climate variability is stationary, e.g. the same patterns of wet and dry days will occur in the future dataset as in the original baseline (Harris et al, 2012). Despite this, it remains a popular approach, given its relative simplicity and low computation demands (e.g. Dacacche et al, 2012). Alternatively, a probabilistic weather generator can be used to generate multiple future time series using perturbed synthetic baselines. Unlike the conventional change factor approach, weather generators are not dependant on the individual having access to a suitably long observed record (Green and Weatherhead, 2013) nor do they assume that the future climate variability is stationary, making them an attractive tool for supporting robust decision making (Groves and Lempert, 2007; Dessai et al, 2009; Lempert and Groves, 2010; Harris et al, 2012). The change factor approach and UKCP09 weather generator (Semenov 2007; Wilks and Wilby, 2009) are both examples of statistical downscaling (Wilby et al, 2004), while they are not utilised here, alternative methods collectively referred to as dynamical downscaling techniques also exist (Mearns et al, 2003). An extensive discussion of the merits and weaknesses of these and other downscaling techniques can be found elsewhere and in greater detail (Prudhomme et al, 2002; Fowler et al, 2007)

The primary source of future climate projections in the UK is the UKCP09 dataset (Murphy, 2009). UKCP09 provides 10,000 probabilistic climate projections at a $25 \mathrm{~km}$ scale resolution generated from 

provided in the format of monthly change factors. Alternatively, daily (and even hourly) projections, and at a finer spatial resolution of $5 \mathrm{~km}^{2}$, are readily available as outputs from UKCP09's weather generator (Jones et al, 2009). The weather generator provides baseline ("control") and future scenario sequences for three different greenhouse gases emission scenarios (low, medium and high) and for selected 30 year time-slices (centred around the 2020s, 2030s, 2040s, 2050s, 2060s, 2070s and 2080 s respectively).

These daily weather datasets can be imported into soil water balance models such as WaSim, freely available via the Cranfield University website, to model the irrigation demand of various crops (Hess and Counsell, 2000). WaSim simulates inflow (infiltration) and outflow (evapotranspiration and drainage) and storage of soil water in response to climate, irrigation and drainage (Depeweg and Fabiola Otero, 2004). WaSim has proven invaluable across a range of previous studies including determining irrigation requirements, optimising water management, assessing the performance of sub-surface drainage systems and studying the effects of climate change on water resources (Depeweg and Fabiola Otero, 2004; Hirekhan et al, 2007, Warren and Holman, 2011). WaSim divides the soil profile into five layers, water moves from upper layers to lower layers when the water content of the respective layer exceeds field capacity. The first three layers are comprised of the surface layer $(0-0.15 \mathrm{~m})$, the active root zone layer (0.15-root depth) and the unsaturated layer below the root zone (root depth-water table). The remaining 2 layers are comprised of the saturated layer above drain depth (water table - drain depth) and the saturated layer below drain depth (depth drain - impermeable layer). The boundary between the second and third layers changes in response to root growth (e.g. in the case of potatoes, layer 2 has zero thickness when root depth is less than $0.15 \mathrm{~m}$, and then increases as the potato develops). Guidance values covering crop can be combined in a cropping pattern (Hess and Counsell, 2000). 
In the field of irrigated agriculture, decision makers have typically relied on the design dry year rule for estimating the volume of irrigation required. A design dry year is defined in the UK as a year with an $80 \%$ probability of non-exceedance (roughly equivalent to the older "fourth driest year of five" rule of thumb). This rule of thumb is generally considered the 'best practice approach' and forms the basis of most water allocation for UK irrigated agriculture (Weatherhead and Knox, 2000).

This study explores the difference between using the change factor approach and the UKCPO9 weather generator for modelling future irrigation demand and informing reservoir design at three sites in the UK. Decision outcomes are obtained using the $80 \%$ probability of non-exceedance rule and an economic optimisation and compared.

Method:

A previous study by Green and Weatherhead (2013) found that the weather generator was reasonably calibrated at a number of UK sites. Three sites representing different agro-climatic conditions distributed around the UK were selected as case studies. These particular sites were chosen because they had the most complete record for the baseline period. Brooms Barn is located in the county of Suffolk, near Bury St Edmunds, approximately 30km east of Cambridge and is the driest of the investigated sites. Slaidburn is located in the district of Lancashire, approximately $60 \mathrm{~km}$ north-west of Leeds and is the wettest site with an average annual rainfall of $1515 \mathrm{~mm}$.year ${ }^{-1}$ for the baseline period. Lastly, Woburn is situated in the county of Bedfordshire, $50 \mathrm{~km}$ north-west of London and is marginally wetter than Brooms barn but with slightly lower annual evapotranspiration. Observed climate data was extracted for the baseline period from the weather station at each site. Additional hydroclimatology data for the baseline period is shown in Table 1. 
Table 1 Weather station sites and records used

\begin{tabular}{|c|c|c|c|c|c|c|c|}
\hline \multirow{2}{*}{ Station } & \multirow{2}{*}{ Lat. } & \multirow{2}{*}{ Long. } & \multirow{2}{*}{$\begin{array}{l}\text { Elevation } \\
\text { (m AOD) }\end{array}$} & \multicolumn{2}{|c|}{ Average annual (1961-1990) } & \multicolumn{2}{|c|}{ Data } \\
\hline & & & & Rain $(\mathrm{mm})$ & $\operatorname{ETo}(\mathrm{mm})$ & From & To \\
\hline Brooms Barn & 52.260 & 0.567 & 75 & 588 & 585 & 1964 & 1990 \\
\hline Slaidburn & 53.987 & -2.433 & 192 & 1515 & 487 & 1961 & 1990 \\
\hline Woburn & 52.014 & -0.595 & 89 & 632 & 564 & 1961 & 1990 \\
\hline
\end{tabular}

162

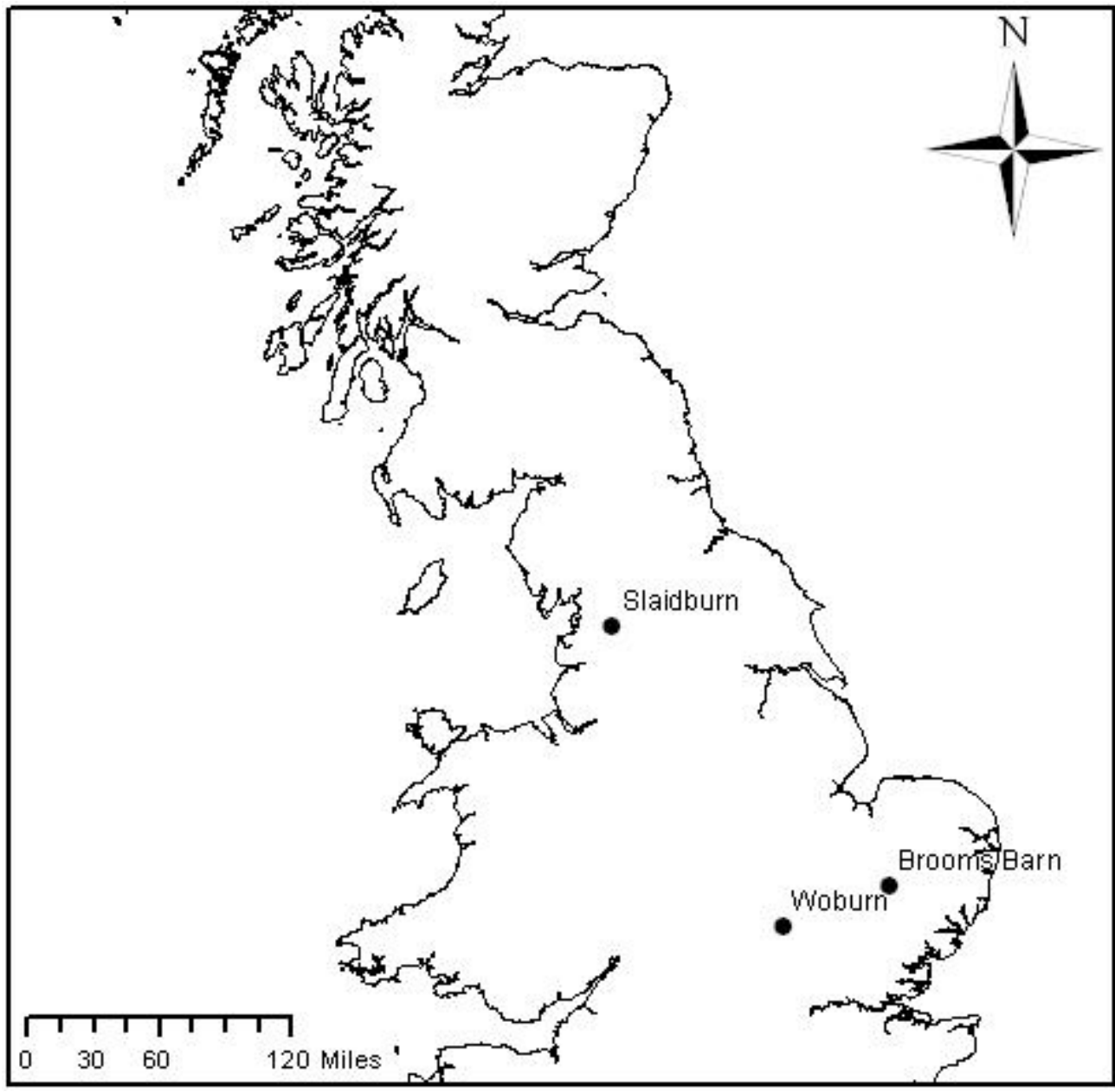

All 10,000 monthly change factor climate projections were extracted from the UKCP09 sample ensemble for the single $25 \mathrm{~km}^{2}$ grid square overlying each weather station, for each emission scenario (i.e. low, medium and high) for the 2050s time slice (i.e. 2040-2069). Baseline evapotranspiration and monthly evapotranspiration change factors were estimated using Penman- 
Monteith (Monteith, 1965); wind speed was assumed to be the same as the observed baseline (1969-1990) due to the lack of earlier baseline data and future projections of wind speed.

Ten thousand climate projections were simultaneously generated using the UKCP09 weather

171 generator, using the same ID codes to allow direct comparison, again for each weather station and 172 each emissions scenario. The UKCP09 weather generator was previously found to be reasonably 173 calibrated at these sites with the exception of some extreme events (which are beyond the scope of our analysis and do not impact the reservoir design) (Green and Weatherhead, 2013).

As the weather generator offers a much greater spatial resolution of $5 \mathrm{~km}^{2}$, data was generated for a grouping of 25 individual grid squares (i.e. a combined area of $25 \mathrm{~km}^{2}$ ) overlying each weather station, to be directly comparable with the 10,000 member ensemble $25 \mathrm{~km}^{2}$ grid square. It should be noted that the weather generator and 10,000 member sample ensemble spatial grids differ slightly in their orientation which may create subtle differences in the projected climate, though because of the large areas used, the impact is considered somewhat negligible. Despite this, the potential impacts on the outcomes of this study are an acknowledged limitation. capable of processing multiple climate files succinctly, so a modified version was developed and employed for this study to read-in multiple climate files and output a single results file containing the daily irrigation demand for each of the 10000 climate files. A potato crop was simulated with a planting depth of $0.15 \mathrm{~m}$, max root depth of $0.7 \mathrm{~m}$ and planting date of $1^{\text {st }}$ April. A rule based irrigation schedule was modelled based on best practice guidelines including scab control (Defra, 2005). This schedule consisted of 4 periods (1 non-irrigation followed by 2 irrigation and 1-non irrigation), applying $15 \mathrm{~mm}$ of water early in the growing season whenever the root zone deficit exceeded $18 \mathrm{~mm}$ during period $2\left(15^{\text {th }}\right.$ May- $30^{\text {th }}$ June $)$ and applying $25 \mathrm{~mm}$ of water whenever the 
blight which can severely reduce the market value of produce (Liu et al 1996). Irrigation is also important for promoting higher tuber numbers, accelerating crop canopy growth, reducing the chance of uneven growth and thumbnail cracking and reducing crop damage during harvesting (Defra, 2005). The soil type was set as sandy loam, which is the dominant soil type for potato crops in England, with an assumed saturation of $43.3 \%$ and field capacity of $24.5 \%$.

The irrigation demand was calculated for each year in the $10,000 \times 30$ year sequences for each site and emission scenario, using both the change factor and weather generator datasets. The values within each sequence were then ranked from smallest to largest. The irrigation demand during the design dry year, (referred to hereafter as $80 \%$ dry year irrigation demand) was calculated for each of the 10,000 sequences, using the $80 \%$ probability of non-exceedance rule. The median, mean, quartile and extreme values for each site, emission scenario and dataset were identified.

For the economic evaluation, typical costs and benefits for clay agricultural reservoirs were obtained from a concurrent study (Weatherhead et al, 2008). The economic benefit of the water contained within each reservoir was calculated on the basis of average water use, assuming an average net benefit (for potatoes) of $£ 1.56 / \mathrm{m}^{3}$ of water used (Morris et al, 1997). Earthwork costs were assumed to be $f 1.125$ per $\mathrm{m}^{3}$ of earth moved, plus an additional $15 \%$ reflecting site investigation costs. $\mathrm{A}$ further $f 20 k$ was added, representing the assumed connection costs of 3-phase electricity. Annual OPEX was assumed to be $1 \%$ of CAPEX, representing the low maintenance cost of clay reservoirs (Weatherhead et al, 2010). Each of the 10,000 sequences was then used to calculate the net present value (NPV) of a range of reservoir sizes, with usable storage capacities equivalent to 0 to $1000 \mathrm{~mm} . \mathrm{year}^{-1}$ for the area irrigated. NPV provides a measure of the present value of the difference between the assumed costs and benefits of a decision. NPV was calculated by discounting the annual net benefit of the reservoir loss OPEX costs with a lumped (non-discounted) CAPEX in year 0, assuming current government discount rate guidelines of $3.5 \%$ on investments of up to 30 years

217 (Treasury HM, 2011). Each reservoir was assumed to last 30 years, representing their typical life 
cycle. The optimum reservoir capacity, defined as the size providing the highest NPV was calculated for each of the 10,000 sequences. The median, mean, quartile and extreme values for each site, emission scenario and dataset were identified as before.

The Mann-Whitney U-test (Mann and Whitney, 1947) was used to establish whether there was significant differences between the change factor and weather generator datasets in terms of both the $80 \%$ dry year irrigation demands and the optimum reservoir capacities. The Mann-Whitney U test was chosen due to the non-parametric nature of the data even after applying transformations. The Mann-Whitney $U$ test is used to test the equality of two population medians. It is considered the non-parametric alternative to the 2-sample t-test, it assumes that the populations are independent and have a similar distribution shape. Unlike the 2-sample t-test it does not require the two populations to be normally distributed.

In addition, a sensitivity analysis was undertaken to establish how sensitive the decision outcome was to the choice of discount rate, benefit of the water and earthwork costs. Each parameter was varied in turn, keeping the other parameters fixed, and the median optimum reservoir capacity identified, calculating the percentage difference before and after varying each parameter. The discount rate was initially fixed at $3.5 \%$, water benefit at $£ 1.56 / \mathrm{m}^{3}$ and earthworks at $£ 1.1 .25 / \mathrm{m}^{3}$, and subsequently scaled up and down using a linear coefficient.

\section{Results and Discussion:}

The $80 \%$ dry year irrigation demands were compared between the change factor and weather generator sequences for each sites and emission scenario (Figure 1). The median $80 \%$ dry year irrigation demand was similar across both datasets. Both also had a similar interquartile and extreme range. These results support the assumption that the weather generator was reasonably calibrated with the observed record (Green and Weatherhead, 2013) and suggest that using the UKCP09 


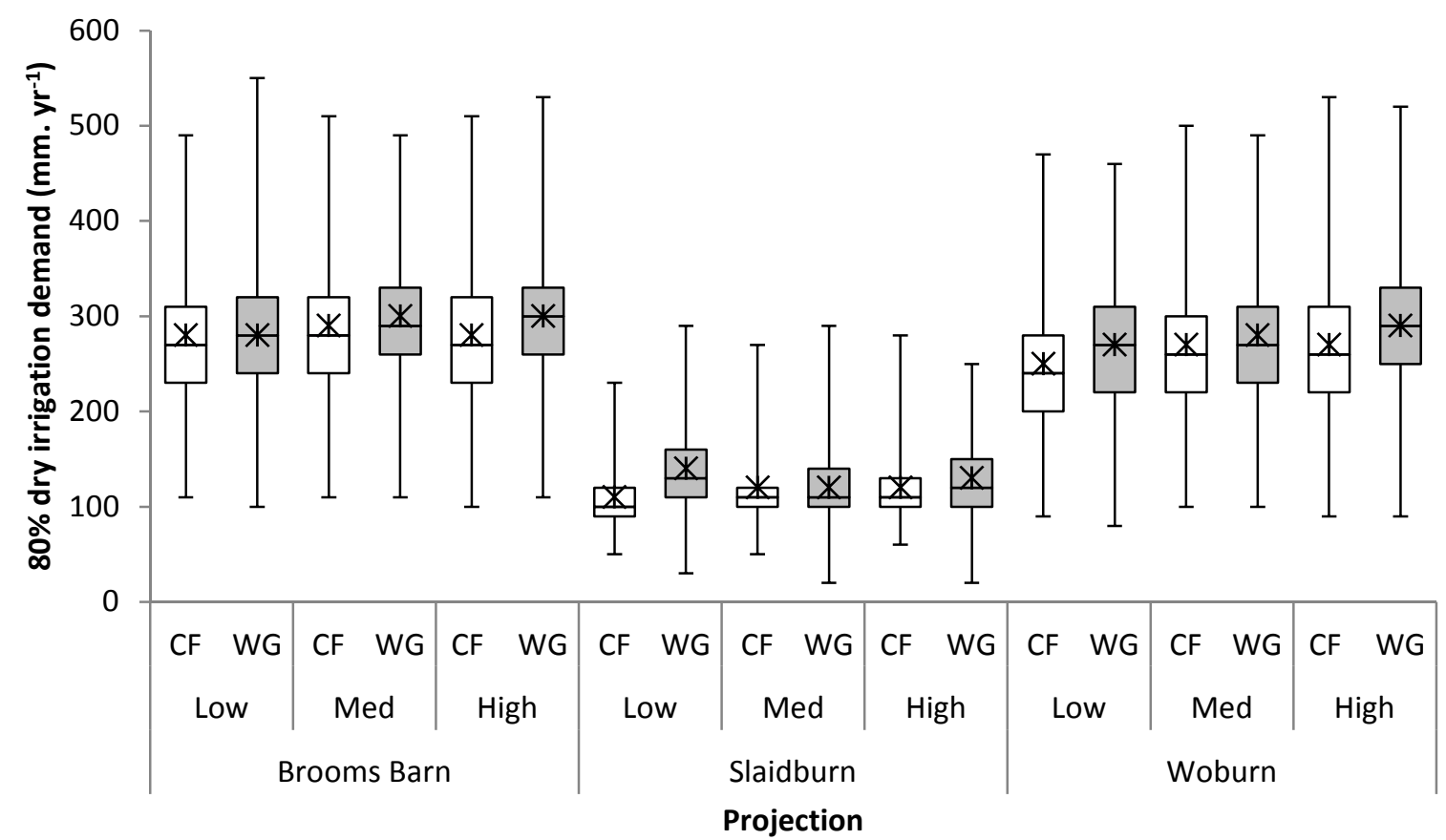

Figure 1. Median (-), mean (X), quartile and extreme values of the $80 \%$ dry year irrigation demand for the change factor (CF) and weather generator (WG) sequences for each site and emission scenario.

Next, the economic performance of various reservoir capacities generated using the full 10000 change factor and weather generator sequences were compared against each other for each site and emission scenario. Figure 2 shows the results obtained for the site of Woburn using the medium emission scenario. Despite subtle differences in the projected NPV, both datasets showed a similar trend in NPV against reservoir capacity. The weather generator projected a higher NPV for most reservoir capacities, based on the median projection, with the exception of small reservoirs with a capacity of less than $100 \mathrm{~mm} . \mathrm{yr}^{-1}$. The NPV range (i.e. the difference between the max payoff and minimum payoff for each reservoir size) is initially quite narrow and increases with reservoir capacity. The NPV range is larger for the weather generator dataset than for the change factor 

optimum reservoir capacity was $340 \mathrm{~mm}$.year ${ }^{-1}$. In contrast, the weather generator estimated the median optimum reservoir capacity to be marginally smaller at $320 \mathrm{~mm}$.year ${ }^{-1}$ but with a $20 \%$ larger NPV. Similar results were recorded for all three emission scenarios for all three sites.

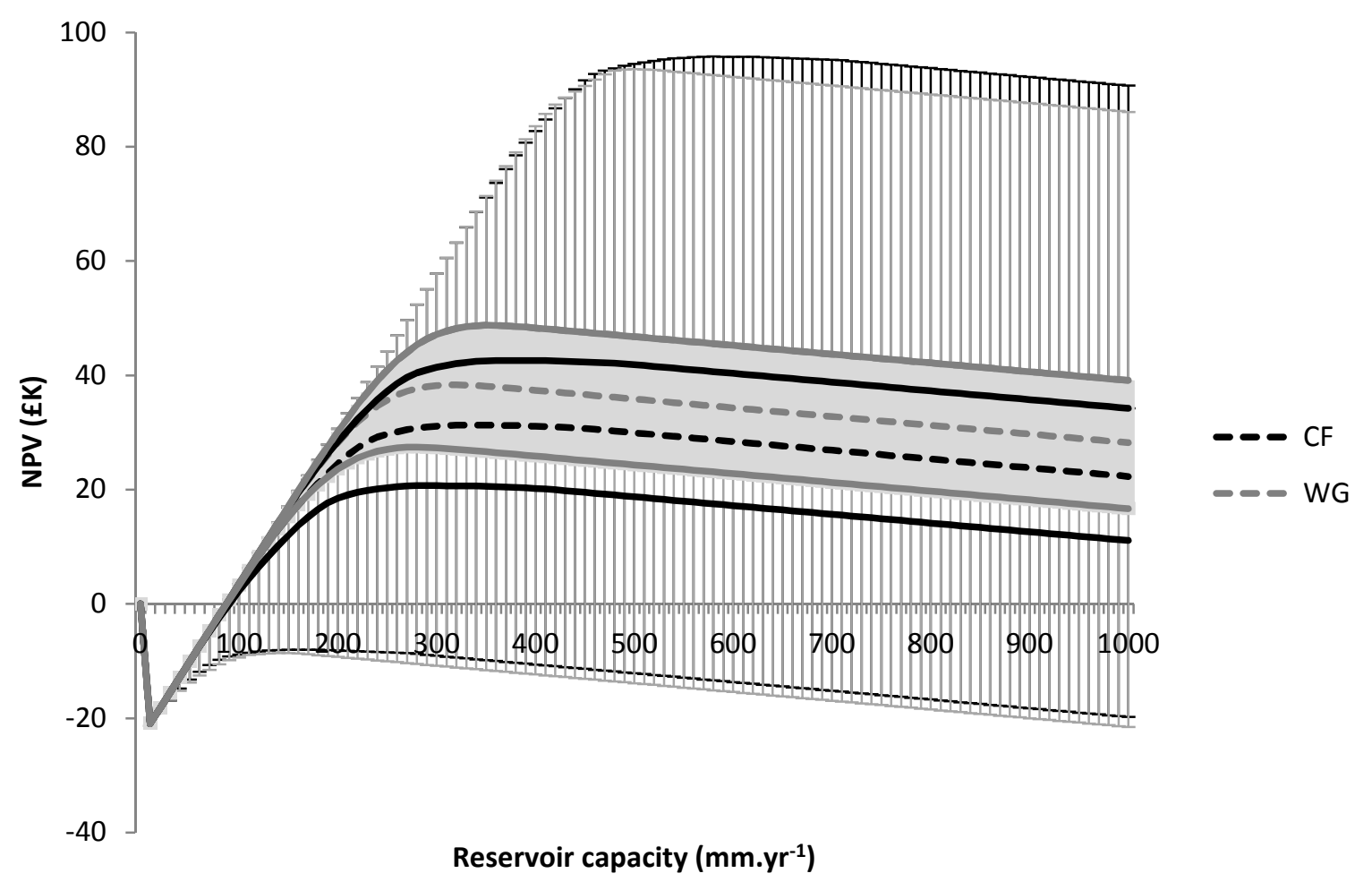

Figure 2. Median, quartile and extreme values of NPV against reservoir capacity for the change factor (CF) and UKCP09 weather generator (WG) sequences for the Woburn site and medium emission scenario.

Statistical analysis was undertaken to establish whether there was significant difference between using the weather generator and change factor datasets in terms of 1) the $80 \%$ dry year irrigation demand and 2) the optimum reservoir capacity. The $80 \%$ dry year irrigation demand values obtained using the weather generator dataset were significantly greater than those from using the change factor dataset. In contrast, the optimum reservoir capacities from the weather generator dataset were significantly lower than from the change factor dataset. However, while the differences were 

generally less than $25 \mathrm{~mm}$.year ${ }^{-1}$, which is only the depth of a typical single application of water. The difference in the optimum reservoir capacities was similarly small (though generally $>25 \mathrm{~mm}$.year ${ }^{-1}$ ), with the exception of the Brooms Barn site. These results again suggest that using the weather generator in place of the conventional change factor, while theoretically leading to more robust decision making, in reality is unlikely to greatly affect the decision outcome.

Table 2. Results of Mann-Whitney U-test statistical analysis comparing $\mathbf{8 0} \%$ dry year irrigation demand and optimum reservoir capacity obtained using economic optimisation with change factor (CF) and weather generator (WG) datasets, showing median reservoir capacity, whether they are significantly different and using 95 confidence interval $(95 \mathrm{Cl})$.

\begin{tabular}{|c|c|c|c|c|c|c|c|c|c|c|c|c|}
\hline \multirow{4}{*}{$\begin{array}{l}\text { Site } \\
\text { Criteria } \\
\text { Emission scen. } \\
\text { Data source }\end{array}$} & \multicolumn{12}{|c|}{ Brooms Barn } \\
\hline & \multicolumn{6}{|c|}{ 80\% Dry year irrigation demand } & \multicolumn{6}{|c|}{ Optimum reservoir capacity } \\
\hline & \multicolumn{2}{|c|}{ Low } & \multicolumn{2}{|c|}{ Med } & \multicolumn{2}{|c|}{ High } & \multicolumn{2}{|c|}{ Low } & \multicolumn{2}{|c|}{ Med } & \multicolumn{2}{|c|}{ High } \\
\hline & $\mathrm{CF}$ & WG & $\mathrm{CF}$ & WG & $\mathrm{CF}$ & WG & $\mathrm{CF}$ & WG & $\mathrm{CF}$ & WG & $\mathrm{CF}$ & WG \\
\hline Res. capacity & 270 & 280 & 280 & 290 & 270 & 300 & 360 & 310 & 370 & 320 & 370 & 330 \\
\hline Sig. difference? & \multicolumn{2}{|c|}{ Yes } & \multicolumn{2}{|c|}{ Yes } & \multicolumn{2}{|c|}{ Yes } & \multicolumn{2}{|c|}{ Yes } & \multicolumn{2}{|c|}{ Yes } & \multicolumn{2}{|c|}{ Yes } \\
\hline P-value (95Cl) & \multicolumn{2}{|c|}{0.00} & \multicolumn{2}{|c|}{0.00} & \multicolumn{2}{|c|}{000} & \multicolumn{2}{|c|}{0.00} & \multicolumn{2}{|c|}{0.00} & \multicolumn{2}{|c|}{0.00} \\
\hline
\end{tabular}

280

\begin{tabular}{|c|c|c|c|c|c|c|c|c|c|c|c|c|}
\hline Site & \multicolumn{12}{|c|}{ Slaidburn } \\
\hline Criteria & \multicolumn{6}{|c|}{$80 \%$ Dry year irrigation demand } & \multicolumn{6}{|c|}{ Optimum reservoir capacity } \\
\hline Emission scen. & \multicolumn{2}{|c|}{$\mathrm{L}$} & \multicolumn{2}{|c|}{ M } & \multicolumn{2}{|c|}{$\mathrm{H}$} & \multicolumn{2}{|c|}{$\mathrm{L}$} & \multicolumn{2}{|c|}{ M } & \multicolumn{2}{|c|}{$\mathrm{H}$} \\
\hline Data & CF & WG & CF & WG & CF & WG & CF & WG & CF & WG & CF & WG \\
\hline Res. capacity & 100 & 130 & 110 & 110 & 110 & 120 & 0 & 0 & 0 & 0 & 0 & 0 \\
\hline Sig. difference? & \multicolumn{2}{|c|}{ Yes } & \multicolumn{2}{|c|}{ Yes } & \multicolumn{2}{|c|}{ Yes } & \multicolumn{2}{|c|}{ Yes } & \multicolumn{2}{|c|}{ Yes } & \multicolumn{2}{|c|}{ Yes } \\
\hline P-value $(95 \mathrm{Cl})$ & \multicolumn{2}{|c|}{0.00} & \multicolumn{2}{|c|}{0.00} & \multicolumn{2}{|c|}{0.00} & \multicolumn{2}{|c|}{0.00} & \multicolumn{2}{|c|}{0.00} & \multicolumn{2}{|c|}{0.00} \\
\hline
\end{tabular}

281

\begin{tabular}{|c|c|c|c|c|c|c|c|c|c|c|c|c|}
\hline \multirow{4}{*}{$\begin{array}{l}\text { Site } \\
\text { Criteria } \\
\text { Emission scen. } \\
\text { Data source }\end{array}$} & \multicolumn{12}{|c|}{ Woburn } \\
\hline & \multicolumn{6}{|c|}{$80 \%$ Dry year irrigation demand } & \multicolumn{6}{|c|}{ Optimum reservoir capacity } \\
\hline & \multicolumn{2}{|c|}{ Low } & \multicolumn{2}{|c|}{ Med } & \multicolumn{2}{|c|}{ High } & \multicolumn{2}{|c|}{ Low } & \multicolumn{2}{|c|}{ Med } & \multicolumn{2}{|c|}{ High } \\
\hline & CF & WG & CF & WG & CF & WG & CF & WG & CF & WG & CF & WG \\
\hline Res. capacity & 240 & 270 & 260 & 270 & 260 & 290 & 320 & 300 & 340 & 320 & 340 & 320 \\
\hline Sig. difference? & \multicolumn{2}{|c|}{ Yes } & \multicolumn{2}{|c|}{ Yes } & \multicolumn{2}{|c|}{ Yes } & \multicolumn{2}{|c|}{ Yes } & \multicolumn{2}{|c|}{ Yes } & \multicolumn{2}{|c|}{ Yes } \\
\hline P-value $(95 \mathrm{Cl})$ & \multicolumn{2}{|c|}{0.00} & \multicolumn{2}{|c|}{0.00} & \multicolumn{2}{|c|}{0.00} & \multicolumn{2}{|c|}{0.00} & \multicolumn{2}{|c|}{0.00} & \multicolumn{2}{|c|}{0.00} \\
\hline
\end{tabular}


Finally, the optimum reservoir capacity was directly compared with the dry year irrigation demand calculated using a range on probability of non-exceedance values $(80 \%, 85 \%, 90 \%, 95 \%$ and $100 \%)$. Based on these initial findings, the $80 \%$ probability of exceedance rule appears to underestimate the optimum reservoir capacity at Brooms Barn and Woburn and overestimate the optimum reservoir capacity at Slaidburn (the wettest site), with a difference of between -120 to $+100 \mathrm{~mm} \cdot$ ha $^{-1}$ (Figure 3 ). The $95 \%$ probability of non-exceedance rule had a smaller difference of between 0 to $+170 \mathrm{~mm}$.year to the optimum reservoir capacity at the sites of Brooms Barn and Woburn. However at the site of

291 Slaidburn, all five probability of non-exceedance rules tested appear to considerably overestimate 292 the optimum reservoir capacity (see Figure 3 ). This result should serve as a warning to those 293 stakeholders who do not consider the underlying economics of their decision; blind use of 294 probability of non-exceedance rules can lead to maladaptation with stakeholders either overdesigning or under-designing their assets. 


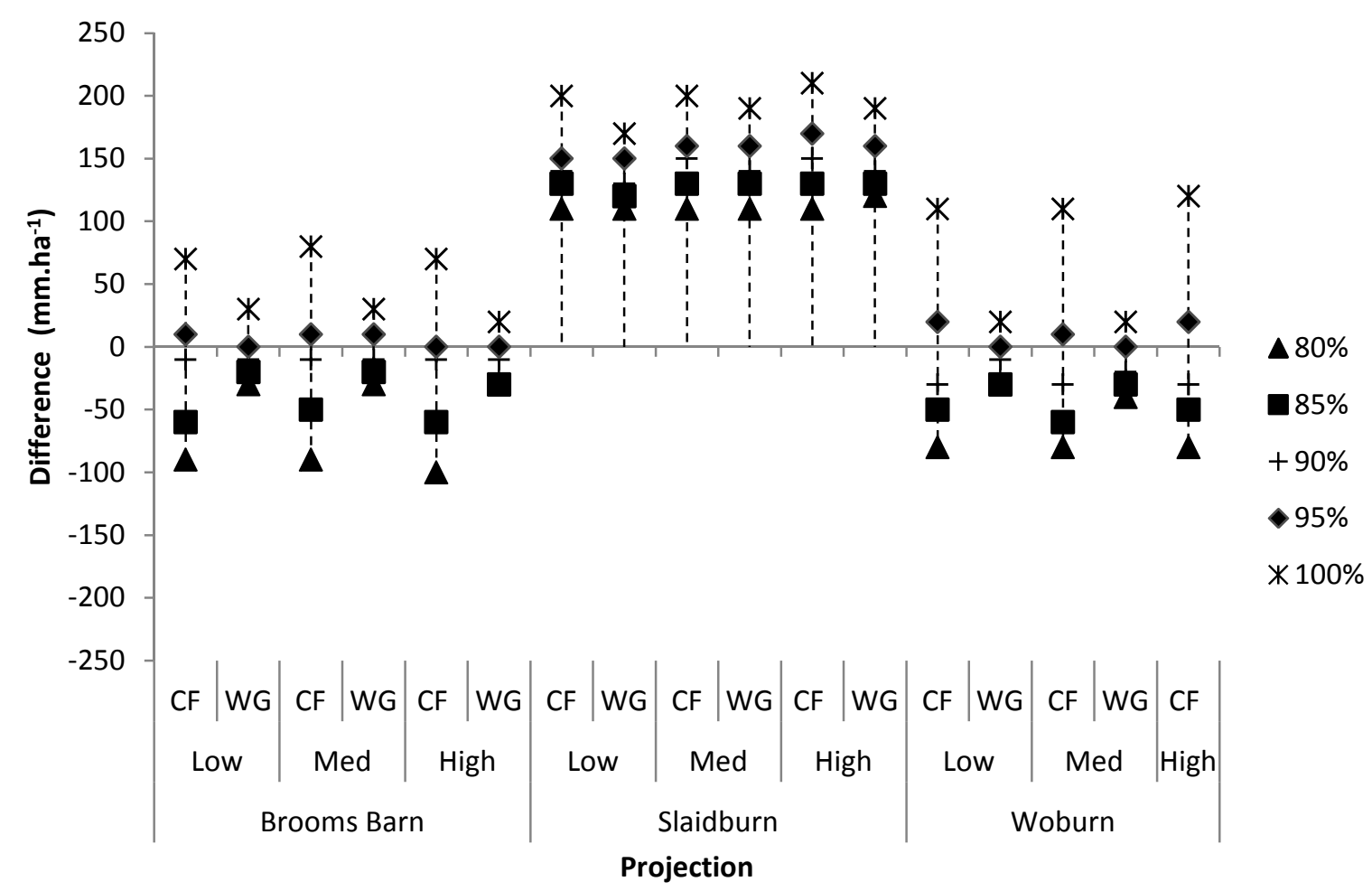

296

Figure 3. Differences between the median dry year irrigation demands using $80 \%$ to $95 \%$ exceedance rules and the median optimum reservoir capacity, for the change factor (CF) and weather generator (WG) sequences for each site and emission scenario.

The results of this study are dependent on several assumptions including 1) discount rate, 2) earth work costs and 3) monetary benefit of the water. Each of these variables is a potential source of uncertainty and may potentially affect the optimum reservoir capacity. As a result, a sensitivity analysis was undertaken to establish whether altering these parameters changed the perceived optimum reservoir capacity.

The sensitivity analysis is presented here for the site of Woburn, for the medium emission scenario and the weather generator dataset. Similar results were obtained for the other sites and emission scenarios and for the change factor dataset. The optimum reservoir capacity was largely insensitive to the discount rate, evident from the near horizontal line, with larger discount rates slightly favouring smaller reservoirs (Figure 4). The reservoir capacity was more sensitive to earthworks 
costs, with larger earthworks costs favouring smaller reservoirs, again as expected. The value of the water in the reservoir had the largest effect on the optimum reservoir capacity; below $f 0.78 . \mathrm{m}^{-3}$ the reservoir produced a negative NPV and was no longer economically viable at this site. Increasing the value of water above $£ 1.56 . \mathrm{m}^{-3}$ had surprisingly little effect on the optimum reservoir capacity, increasing it by only $9.7 \%$ even up to a value of $£ 4.68 . \mathrm{m}^{-3}$; this reflects the point that useful capacity is limited by demand, with decreasing returns to additional capacity.

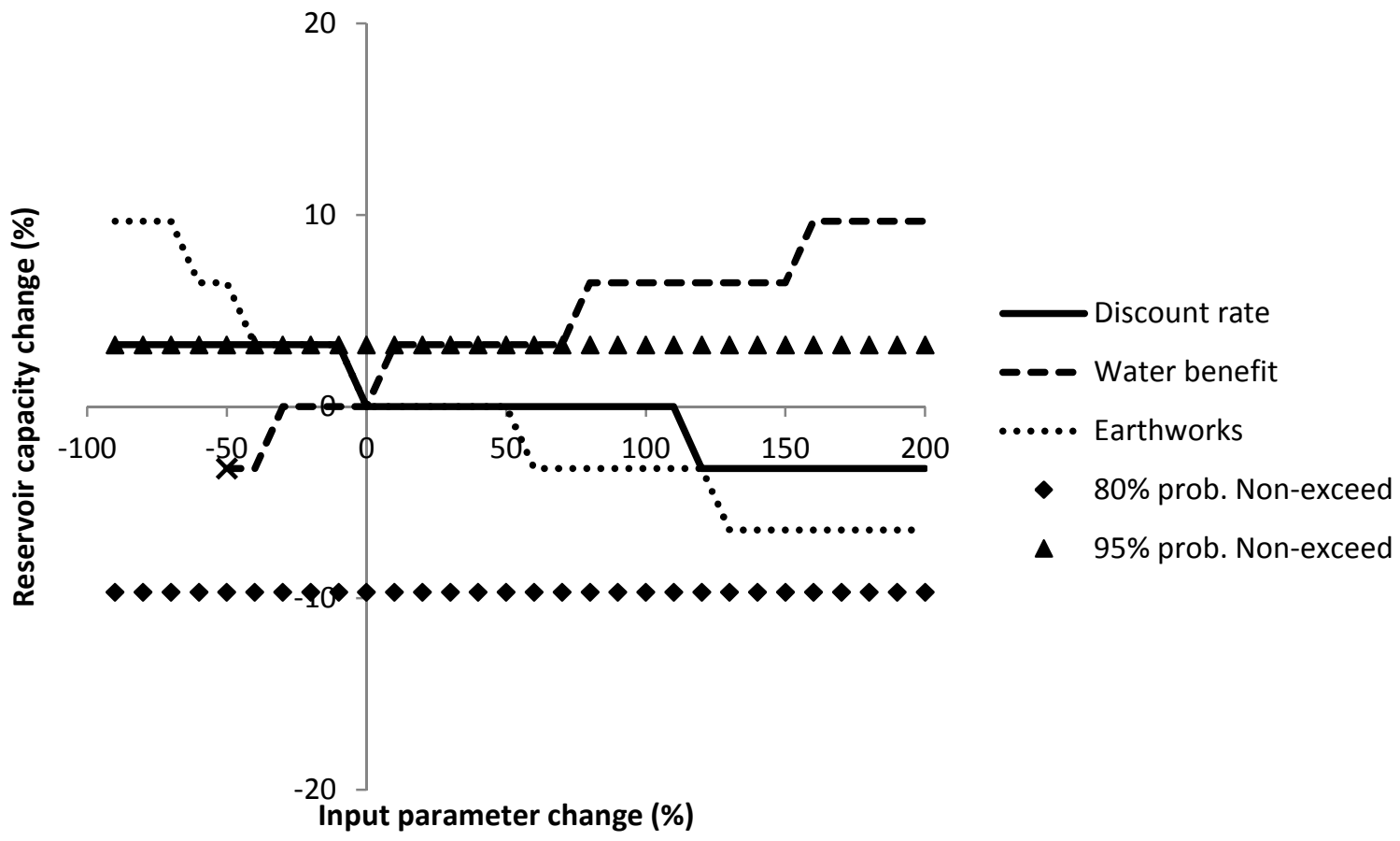

Figure 4. Sensitivity analysis comparing optimum reservoir capacity against discount rate, water benefit and earthworks cost, showing changes relative to base parameter values, for the Woburn site and medium emission scenario. The $\mathbf{8 0 \%}$ and $95 \%$ dry year irrigation demands are also shown for comparison.

These variations in median optimum reservoir capacity were subsequently compared to the capacities given by the simpler $\%$ exceedance rules, in this case the $80 \%$ and $95 \%$ dry year irrigation demand. For the Woburn site and the base variable values, the $95 \%$ probability of non-exceedance rule out performs the $80 \%$ probability of non-exceedance rule (Figure 4). At larger discount rates $(>7 \%)$ the $80 \%$ rule works better, and for lower earthwork costs (less than $f 1.80 . \mathrm{m}^{-3}$ ) the two rules 
are equally close. For all water values, the $95 \%$ probability of non-exceedance rule was nearer the optimum value, but both rules failed to show that the reservoir was no longer economically viable when the water value was less than $f 0.78 . \mathrm{m}^{-3}$. More case studies would be needed to confirm theses are general results, but they suggest that the $80 \%$ rule may be misleading.

It should be noted that these findings are conditional on the view that the median optimum reservoir capacity of the 10,000 sequences represents the most appropriate course of action (akin to the 'Laplacian' view of investment appraisal) (French, 1986). Decision makers who are particularly risk averse or risk seeking may disagree with this assumption and may instead use the quartile or even best/worst case projections, though for the vast majority of stakeholders our stated assumptions should suffice.

Global climate models (GCM) providing "high" resolution daily projections are few in number and those which do are considered less accurate (Paluktikof et al, 1997; Huth et al, 2001). As a result, GCM climate change projections often need to be downscaled both spatially and temporally before they can be of any use for decision makers. Numerous downscaling approaches are available, including but not limited to the change factor approach and UKCP09 weather generator considered here. Different downscaling techniques come with their own advantages and disadvantages; see Wilby et al (2004) and Fowler et al (2007) for extensive reviews. The UKCP09 weather generator is theoretically better than the conventional change factor approach, given that it allows for nonstationary variability to be simulated and thus incorporated into climate change risk assessments and adaptation planning (Harris et al, 2012). The UKCP09 weather is however not without its flaws, a previous study by Tham et al (2011) found that the weather generator initially released with UKCP09 was unable to reproduce observations of key climate variables including sunshine duration and solar irradiation. generator to improve its predictive capabilities, which were later verified by Eames et al, (2012). 
351 They found that the weather generator was capable of producing weather data that was consistent

352 with historical monthly observations of wind, speed, direct irradiation, diffuse irradiation, global irradiation, maximum temperature, minimum temperature and mean temperature. This result is consistent with previous findings by Green and Weatherhead (2013) which showed that the UKCP09 was capable of reproducing observed precipitation and evapotranspiration and annual irrigation demand reasonably well. Eames et al (2012) also noted that subsequent iterations of the UKCP09 weather generator had issues reproducing a realistic distribution of sunshine hours and direct and diffuse irradiation which can lead to absurd conclusions. We expect that the UKCP09 weather generator will be gradually improved over time to reduce or remove these concerns; while they did not affect the findings of this study they may have implications for other applications where hourly data is of high importance.

A criticism of the change factor method, as previously noted, is that it assumes that the temporal and spatial structure of future precipitation and evapotranspiration remains unchanged (Diaz-Nieto and Wilby, 2005; Fowler et al, 2005; Minvlle et al, 2008; Harris et al, 2012). In some situations, it is necessary to evaluate changes in climate variability and not just changes in means (Semenov et al, 1998). Despite this, the change factor approach remains popular because of its simplicity and is useful for converting monthly change factors into daily projections needed to model most hydrological processes without incurring excessive expense (Minville et al, 2008).

\section{Conclusions}

This study found that use of a weather generator not greatly alter the decision outcome compared to using the conventional and relative crude change factor approach, suggesting that the changes in day-to-day climate variability that is simulated by the weather generator are not significant enough to warrant action when informing irrigation reservoir design. This result is contrary to the expectation that the UKCP09 weather generator lends itself to more robust decision making; in reality the difference between the two approaches is negligible. 
The core benefits of the weather generator may continue to make it an attractive tool to use, those being that it provides hourly climate data and readily available evapotranspiration data. Whether these benefits outweigh its fundamental limitations including the poor simulation of extreme meteorological events, is subject to the sensitivity of each application and the user's requirements. The study also found that the "best-practice" approach of using the $80 \%$ probability of nonexceedance rule is inadequate and designers should instead investigate the fundamental economics (e.g. NPV) that underpin the decision making process.

\section{Acknowledgements}

We would like to thank the Engineering and Physical Sciences Research Council (EPSRC) and HR Wallingford for funding this research.

\section{References}

Adger, W. N., Arnell, N. W. \& Tompkins, E. L. 2005 Adapting to climate change: perspectives across scales. Global Environmental Change Part A. 15, 75-76.

Anderson, K. \& Bows, A. 2011 Beyond 'dangerous' climate change: emission scenarios for a new world. Philosophical Transactions of the Royal Society A: Mathematical, Physical and Engineering Sciences. 369, 20-44.

Brown, C. \& Wilby, R. L. 2012 An alternate approach to assessing climate risks. EOS Transactions American Geophysical Union. 93, 401-402.

Daccache, A., Keay, C., Jones, R. J. A., Weatherhead, E. K., Stalham, M. A. \& Knox, J. W. 2012 Climate change and land suitability for potato production in England and Wales: impacts and adaptation. Journal of Agricultural Science. 150, 161-177.

Defra. 2005 Irrigation Best Practice - A Guide for Potato Growers, Department for Environment, Food and Rural Affairs (Horticulture and Potatoes Division).

Depeweg, H. \& Fabiola Otero, M. 2004 Optimization of water management in the RUT Irrigation District, Colombia. Irrigation and drainage. 53, 55-67.

Dessai, S., Hulme, M., Lempert, R. \& Pielke Jr, R. 2009 Climate prediction: a limit to adaptation. Adapting to climate change: thresholds, values, governance, In: Adapting to Climate Change (N. W. Adger, I. Lorenzone, K. L. O’brien eds.) Cambridge University Press, Cambridge, UK, pp. 64-78.

Dessai, S., Lu, X. \& Risbey, J. S. 2005 On the role of climate scenarios for adaptation planning. Global Environmental Change. 15, 87-97. 
Diaz-Nieto, J. \& Wilby, R. L. 2005 A comparison of statistical downscaling and climate change factor methods: impacts on low flows in the River Thames, United Kingdom. Climatic Change. 69, 245-268.

Eames, M., Kershaw, T. \& Coley, D. 2012 A comparison of future weather created from morphed observed weather and created by a weather generator. Building and Environment. 56, 252-264.

Fowler, H. J., Kilsby, G. C., O'Connell, P. E. \& Burton, A. 2005 A weather-type conditioned multi-site stochastic rainfall model for the generation of scenarios of climatic variability and change. Journal of Hydrology. 308, 50-66.

Fowler, H., Blenkinsop, S. \& Tebaldi, C. 2007 Linking climate change modelling to impacts studies: recent advances in downscaling techniques for hydrological modelling. International Journal of Climatology. 27, 1547-1578.

French, S. 1986 Decision theory: an introduction to the mathematics of rationality, Halsted Press, New York.

Fung, F., Lopez, A. \& New, M. 2011 Water availability in 2 C and 4 C worlds. Philosophical transactions of the Royal Society A: mathematical, physical and engineering sciences. 369, 99-116.

Füssel, H. 2007 Adaptation planning for climate change: Concepts, assessment approaches, and key lessons. Sustainability Science. 2, 265-275.

Green, M. \& Weatherhead, E. K. 2013 Irrigation demand modelling using the UKCP09 weather generator: Lessons learned. Journal of Water and Climate Change. Under review.

Groves, D. G. \& Lempert, R. J. 2007 A new analytic method for finding policy-relevant scenarios. Global Environmental Change. 17, 73-85.

Harris, C. N. P., Quinn, A. D. \& Bridgeman, J. 2012 The use of probabilistic weather generator information for climate change adaptation in the UK water sector. Meteorological Applications.

Hedger, M. M., Connell, R. \& Bramwell, P. 2006 Bridging the gap: Empowering decision-making for adaptation through the UK Climate Impacts Programme. Climate Policy. 6, 201-215.

Hess, T. M. \& Counsell, C. 2000 A water balance simulation model for teaching and learning-WaSim. ICID British Section Irrigation and drainage research day. 29, 1204-1213.

Hirekhan, M., Gupta, S. \& Mishra, K. 2007 Application of WaSim to assess performance of a subsurface drainage system under semi-arid monsoon climate. Agricultural Water Management. 88, 224-234.

Holman, I. P., Tascone, T. M. \& Hess, T. M. 2009 A comparison of stochastic and deterministic methods for modelling potential groundwater recharge under climate change in East Anglia, UK: implications for groundwater resource management. Hydrogeology Journal. 17, 1629-1641.

Huth, R., Kysely, J. \& Dubrovsky, M. 2001 Time structure of observed, GCM-simulated, downscaled, and stochastically generator daily temperature series. Journal of Climate. 20, 4047-4061. 
440 IPCC. 2001 Climate Change 2001: Synthesis Report. A Contribution of Working Groups I, II, III to the 441 Third Assessment Report of the Intergovernmental Panel on Climate Change. R.T. Watson and the 442 Core Team eds. Cambridge University Press, Cambridge and New York, 398 pp.

443 Jones, P., Kilsby, C., Harpham, C., Glenis, V. \& Burton, A. 2009 UK Climate Projections science report: 444 Projections of future daily climate for the UK from the Weather Generator. University of Newcastle, 445 UK.

446 Kang, Y., Khan, S. \& Ma, X. 2009 Climate change impacts on crop yield, crop water productivity and

food security - A review. Progress in Natural Science. 19, 1665-1674.

Knox, J. W., Rodriguez-Diaz, J. A., Weatherhead, E. K. \& Kay, M. G. 2010 Development of a water-use strategy for horticulture in England and Wales - A case study. Journal of Horticultural Science and Biotechnology. 85, 89-93.

Lempert, R. J. \& Groves, D. G. 2010 Identifying and evaluating robust adaptive policy responses to climate change for water management agencies in the American west. Technological Forecasting and Social Change. 77, 960-974.

Liu, D., Anderson, N. A. \& Kinkel, L. L. 1996 Selection and characterization of strains of Streptomyces suppressive to the potato scab pathogen. Canadian journal of microbiology. 42, 487-502.

Loaiciga, H. A., Maidment, D. \& Valdes, J. B. 2000 Climate change impacts in a regional karst aquifer,

Minville, M., Brissette, F. \& Leconte, R. 2008 Uncertainty of the impact of climate change on the Mann, H. B. \& Whitney, D. R. 1947 On a Test of Whether one of Two Random Variables is Stochastically Larger than the Other. Annals of Mathematical Statistics. 18, 50-60.

Mearns, L. O., Giorgi, F., Whetton, P., Pabon, D., Hulme, M. \& Lal, M. 2003 Guidelines for use of climate scenarios developed from regional climate model experiments. Data Distribution Centre of the Intergovernmental Panel on Climate Change. Available from http://www.ipccdata.org/guidelines/dgm no1 v1 10-2003.pdf.

Moser, S. C. \& Ekstrom, J. A. 2010 A framework to diagnose barriers to climate change adaptation.

475 Parry, M. L. 2007 Climate Change 2007: Impacts, Adaptation and Vulnerability: Working Group I 476 Contribution to the Fourth Assessment Report of the IPCC, Cambridge University Press. 
477 Prudhomme, C., Reynard, N. \& Crooks, S. 2002 Downscaling of global climate models for flood 478 frequency analysis: Where are we now?. Hydrological Processors. 16, 1137-1150.

479 Ranger, N., Millner, A., Dietz, S., Fankhauser, S., Lopez, A. \& Ruta, G. 2010 Adaptation in the UK: a 480 decision-making process, Environment Agency.

481 Sanderson, M., Hemming, D. \& Betts, R. 2011 Regional temperature and precipitation changes under 482 high-end ( $\geq 4$ C) global warming. Philosophical Transactions of the Royal Society A: Mathematical, 483 Physical and Engineering Sciences. 369, 85-98.

484 Semenov, M. A. 2007 Development of high-resolution UKCIP02-based climate change scenarios in 485 the UK. Agricultural and Forest Meteorology. 144, 127-138.

486 Tang, S. \& Dessai, S. 2012 Usable science? The UK Climate Projections 2009 and decision support for 487 adaptation planning. Weather, Climate, and Society. 4, 300-313.

Tham, Y., Muneer, T., Levermore, G. J. \& Chow, D. 2011 An examination of UKCIP02 and UKCP09 solar radiation data sets for the UK climate related to their use in building design. Building Services Engineering Research and Technology. 32, 207-228. Observed adaptation to climate change: UK evidence of transition to a well-adapting society. Global Environmental Change. 20, 627-635.

Treasury, H. M. 2003 The Green Book: appraisal and evaluation in central government: Treasury guidance, Stationery Office.

Warren, A. J. \& Holman, I. P. 2012 Evaluating the effects of climate change on the water resources for the city of Birmingham, UK. Water and Environment Journal. 26, 361-370.

Weatherhead, E. K. \& Knox, J. W. 2000 Predicting and mapping the future demand for irrigation water in England and Wales. Agricultural Water Management. 43, 203-218.

500 Weatherhead E. K, Nox J. W, and Kay M. G. 2008 Thinking about an irrigation reservoir? Technical of climate scenarios developed from statistical downscaling methods, Supporting material of the Intergovernmental Panel on Climate Change, available from the DDC of IPCC TGCIA, 27.

Wilks D. S. \& Wilby, R. L. 1999 The weather generator game: a review of stochastic weather models. Progress in Physical Geography. 23, 329-357.

507 Winkler, J. A., Palutikof, J. P., Goodess, C. M. \& Andresen, J. A. 1997 The simulation of daily 508 temperature time series from GCM Output. Part 1: comparison of model data with observations. 509 Journal of Climate. 10, 2497-2513. 